\title{
Erratum: Many-Body Models for Molecular Nanomagnets [Phys. Rev. Lett. 110, 157204 (2013)]
}

\author{
A. Chiesa, S. Carretta, P. Santini, G. Amoretti, and E. Pavarini
}

(Q) (Received 20 January 2021; published 12 February 2021)

DOI: 10.1103/PhysRevLett.126.069901

We noticed three misprints in the Letter. First, in Eq. (1) the operator $c_{i^{\prime} m^{\prime} \sigma}^{\dagger}$ should be replaced with $c_{i^{\prime} m^{\prime} \sigma}$. The correct Hamiltonian is

$$
\begin{aligned}
H= & -\sum_{i i^{\prime}} \sum_{\sigma} \sum_{m m^{\prime}} t_{m, m^{\prime}}^{i, i^{\prime}} c_{i m \sigma}^{\dagger} c_{i^{\prime} m^{\prime} \sigma} \\
& +\frac{1}{2} \sum_{i i^{\prime}} \sum_{\sigma \sigma^{\prime}} \sum_{m m^{\prime}} \sum_{p p^{\prime}} U_{m p m^{\prime} p^{\prime}}^{i, i^{\prime}} c_{i m \sigma}^{\dagger} c_{i^{\prime} p \sigma^{\prime}}^{\dagger} c_{i p^{\prime} \sigma^{\prime}} c_{i^{\prime} m^{\prime} \sigma} \\
& +\sum_{i} \lambda_{i} \mathbf{S}_{i} \cdot \mathbf{L}_{i}-H_{\mathrm{DC}} .
\end{aligned}
$$

Next, the term $\left|t_{n, n^{\prime}}^{i, i^{\prime}}\right|^{2}$ is missing in the numerator of the second line of Eq. (3), which should read

$$
\begin{aligned}
\Gamma_{\mathrm{SE}}^{i, i^{\prime}} \sim & \frac{2}{9} \sum_{n^{\prime}=1}^{3} \sum_{n=4}^{5} \frac{\left|t_{n^{\prime}, n}^{i, i^{\prime}}\right|^{2}+\left|t_{n, n^{\prime}}^{i, i^{\prime}}\right|^{2}}{U+\varepsilon_{n}-\varepsilon_{n^{\prime}}} \\
& +\frac{2}{9} \sum_{n^{\prime}=1}^{3} \sum_{n=1}^{3} \frac{\left|t_{n^{\prime}, n}^{i, i^{\prime}}\right|^{2}+\left|t_{n, n^{\prime}}\right|^{2}}{U+2 J+\varepsilon_{n}-\varepsilon_{n^{\prime}}} \\
& -\frac{2}{9} \sum_{n^{\prime}=1}^{3} \sum_{n=4}^{5} \frac{\left|t_{n^{\prime}, n}^{i, i^{\prime}}\right|^{2}+\left|t_{n, n^{\prime}}^{i, i^{\prime}}\right|^{2}}{U-3 J+\varepsilon_{n}-\varepsilon_{n^{\prime}}} .
\end{aligned}
$$

Last, the hopping integral $t_{1,1}^{1,2}$ in Table $\mathrm{I}$ is $-0.131 \mathrm{eV}$. In actual calculations, the correct value of $t_{1,1}^{1,2}$ and the exact superexchange formula were used. Equation (2) was only introduced to discuss qualitatively the superexchange mechanism. Therefore the misprints pointed out do not affect the effective spin-Hamiltonian derived.

We would like to thank G. Kamieniarz, R. Lemanski, and J. Matsiak for drawing our attention to these missprints. 